\title{
Uso do Clodronato Endovenoso na Calcinose Difusa em uma Criança com Síndrome de Superposição Esclerose Sistêmica e Dermatomiosite ${ }^{(*)}$
}

\section{Use of Clodronate in Extensive Calcinosis in a Child with Systemic Sclerosis and Dermatomyositis Overlap}

\author{
Gláucio R. Werner de Castro ${ }^{(1)}$, Percival D. Sampaio-Barros ${ }^{(2)}$, Simone Appenzeller ${ }^{(3)}$, \\ João Francisco Marques Neto ${ }^{(4)}$ e Adil M. Samara ${ }^{(4)}$
}

\section{RESUMO}

Os autores descrevem o caso de uma garota negra, com diagnóstico de síndrome de superposição dermatomiosite e esclerose sistêmica, que desenvolveu calcinose difusa, complicada por infecções secundárias e significativa limitação funcional de membros. Tratamento com colchicina, diltiazem e alendronato sódico não se mostrou eficaz no controle da calcinose, requerendo uso endovenoso bimestral de clodronato, que contribuiu para significativa melhora na cicatrização das úlceras cutâneas e na qualidade de vida.

Palavras-chave: esclerose sistêmica, dermatomiosite, calcinose, clodronato.

\section{INTRODUÇÃO}

A calcinose é uma manifestação comum de várias doenças difusas do tecido conjuntivo (DTC), particularmente esclerose sistêmica (ES) e dermatomiosite (DM). Na ES, a calcinose freqüentemente se desenvolve em pacientes com ES limitada, ou ES difusa em fase tardia ${ }^{(1)}$. Estes depósitos ocorrem predominantemente nas polpas digitais e nos tecidos periarticulares, mas também podem afetar sítios de traumas de repetição e evoluir com a formação de extensas

\begin{abstract}
The authors report an Afro-Brazilian girl with systemic sclerosis and dermatomyositis overlap who evolved with extensive calcinosis, complicated by secondary infections and important disability. Treatment with colchicine, diltiazem and sodium alendronate was not effective in the control of calcinosis, requiring the use of bimonthly intravenous doses of clodronate that resulted in significant improvement in the healing of cutaneous ulcers and in the quality of life of the patient.
\end{abstract}

Keywords: systemic sclerosis, dermatomyositis, calcinosis, clodronate.

massas. A calcinose costuma acometer casos crônicos de DM, especialmente aqueles que se iniciam na infância, e é rara na doença do adulto ${ }^{(2)}$; há predileção por áreas de microtraumas de repetição. A calcinose pode causar significativa incapacidade funcional em conseqüência da dor, contraturas articulares, úlceras cutâneas e atrofia muscular; os problemas estéticos ocasionados podem acarretar traumas sociais e psicológicos. Seu tratamento freqüentemente é desapontador, uma vez que, até o momento, nenhuma droga testada produziu uma melhora consistente.

\footnotetext{
* Trabalho realizado na Disciplina de Reumatologia do Departamento de Clínica Médica da Faculdade de Ciências Médicas da Universidade Estadual de Campinas (FCM-UNICAMP). Recebido em 06/10/03. Aprovado, após revisão, em 16/01/04.

1. Ex-Residente. FCM-UNICAMP.

2. Assistente-Doutor. FCM-UNICAMP.

3. Reumatologista-Assistente. FCM-UNICAMP.

4. Professor Titular. FCM-UNICAMP.
}

Endereço para correspondência: Dr. Percival D. Sampaio-Barros. Disciplina de Reumatologia, Departamento de Clínica Médica. Faculdade de Ciências Médicas Universidade Estadual de Campinas (FCM-UNICAMP). Barão Geraldo, Campinas, SP, CEP 13081-970. E-mail: psbarros@fcm.unicamp.br 


\section{RELATO DO CASO}

Em abril de 1998, uma criança negra do sexo feminino, com oito anos de idade, foi encaminhada ao ambulatório de Esclerodermia de nossa instituição. Ela referia uma história de um ano de espessamento cutâneo progressivo, associado a fenômeno de Raynaud, poliartralgia inflamatória, fraqueza muscular proximal e disfagia. $\mathrm{Na}$ avaliação inicial, apresentava endurecimento difuso da pele (escore cutâneo de Rodnan modificado $=28$ ); não apresentava calcinose, telangiectasias ou distúrbios de pigmentação. $\mathrm{O}$ exame físico do sistema musculoesquelético mostrava fraqueza muscular de predomínio proximal, sem artrite associada. As avaliações cardíaca, pulmonar, abdominal e neurológica eram normais. Exames laboratoriais mostraram hemograma, função renal e hepática normais, com aumento dos níveis séricos de creatinofosfoquinase (1842). Fator antinuclear (FAN), anticorpo anti-centrômero, anti-Scl 70 e anti-Jo1 foram negativos. A radiografia das mãos revelou acrosteólise (reabsorção das extremidades das falanges distais) (figura 1). Radiografia de tórax, eletrocardiograma, ecocardiograma com Doppler e esofagograma contrastado também foram normais. A paciente não permitiu a realização da eletroneuromiografia. Foi feito o diagnóstico de ES associada à DM, sendo iniciado tratamento com metotrexato $(5 \mathrm{mg} / \mathrm{semana}$, via oral), diltiazem $(60 \mathrm{mg} / \mathrm{dia})$, naproxeno $(500 \mathrm{mg} / \mathrm{dia})$ e deflazacort $(6 \mathrm{mg} / \mathrm{dia})$. Após um ano de tratamento, a paciente apresentou normalização da fraqueza muscular e dos níveis séricos de CPK, com melhora progressiva do espessamento cutâneo.

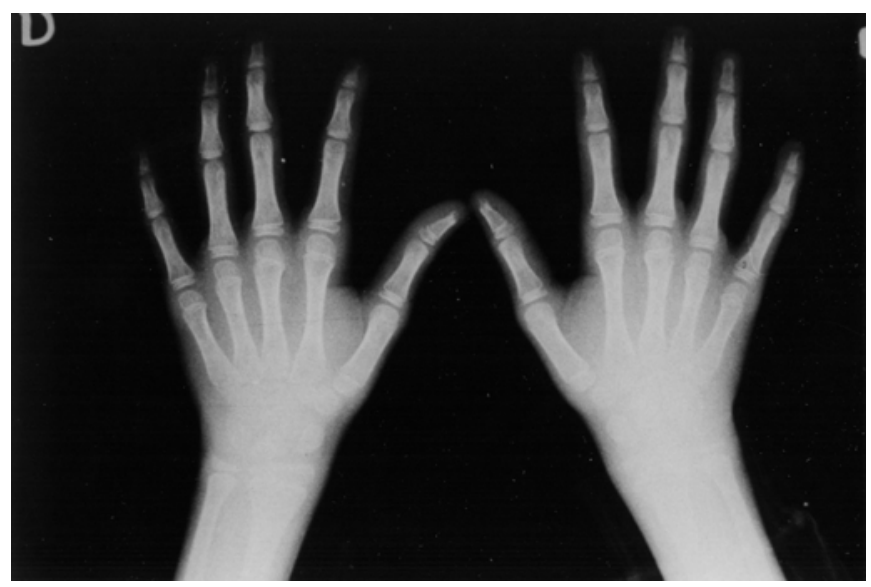

FIGURA 1 - Radiografia das mãos: reabsorção das extremidades das falanges distais (acrosteólise); aspecto radiológico característico da esclerose sistêmica.
Em setembro de 1999, a paciente passou a se queixar da formação de numerosos nódulos subcutâneos, indolores e móveis, acometendo antebraços, coxas, joelhos e nádegas, diagnosticados como calcinose à radiografia das áreas afetadas. Foi então iniciado tratamento com colchicina, na dose de $0.5 \mathrm{mg} / \mathrm{dia}$, que causou diarréia; a dose do diltiazem foi então aumentada para $120 \mathrm{mg} /$ dia mas, após um ano, os nódulos ainda apresentavam crescimento lento no seu número e tamanho, resultando em úlceras cutâneas recorrentes e causando dor significativa e importante limitação em suas atividades de vida diária (figuras 2 e 3). Estas úlceras cutâneas freqüentemente apresentavam infecção bacteriana secundária, requerendo uso de antibióticos. O seguimento radiológico demonstrou aumento no tamanho e na extensão da calcinose (figuras 4 e 5). Alendronato sódico em dose diária de $10 \mathrm{mg}$ foi introduzido em fevereiro de 2001, sendo progressivamente aumentado para $40 \mathrm{mg} / \mathrm{dia}$, mas não resultou em nenhuma melhora após nove meses de uso, sendo suspenso em novembro de 2001.

Desta forma, decidiu-se iniciar o uso de clodronato endovenoso, na dose bimestral de $10 \mathrm{mg} / \mathrm{kg}$. Após a primeira infusão, houve cicatrização das úlceras cutâneas. As infusões subseqüentes resultaram em amolecimento e redução de tamanho dos nódulos subcutâneos. Após um ano de tratamento, a paciente não apresentou recorrência das úlceras cutâneas, e não cursou com nenhum efeito adverso, incluindo hipocalcemia pós-infusão. A despeito da melhora clínica, a comparação das radiografias e cintilografias ósseas pré e pós-clodronato não evidenciou alterações nem no número nem no tamanho das lesões.

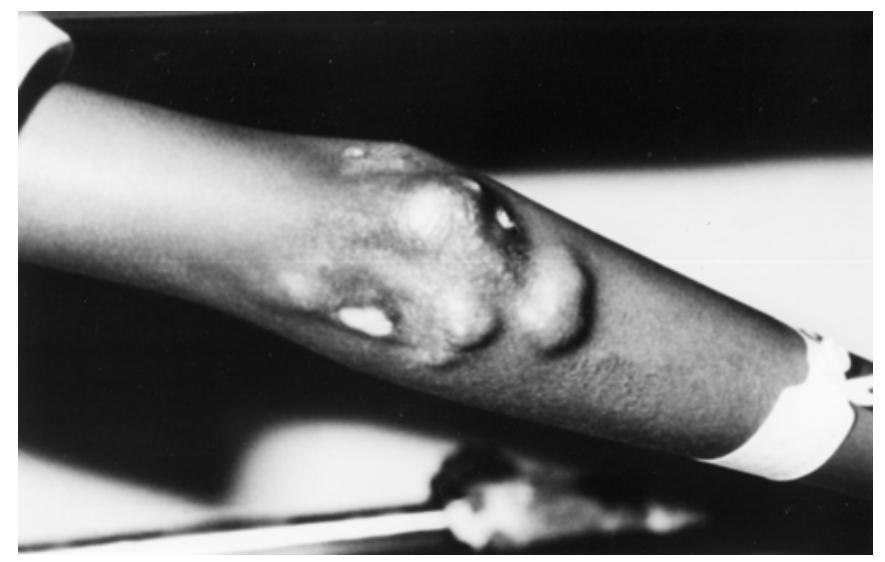

FiguRA 2 - Úlceras cutâneas - superfície extensora dos cotovelos. 


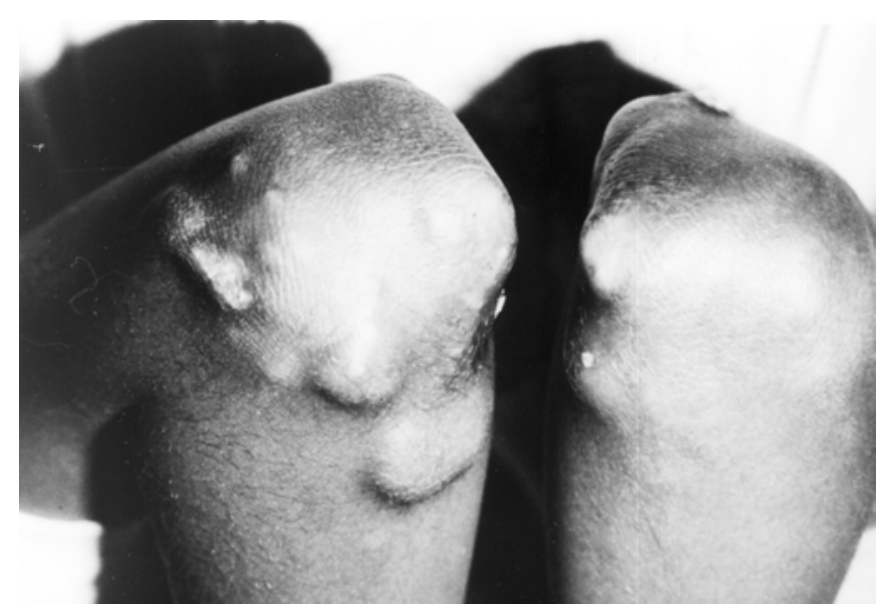

FiguRA 3 - Úlceras cutâneas - superfície extensora dos joelhos.

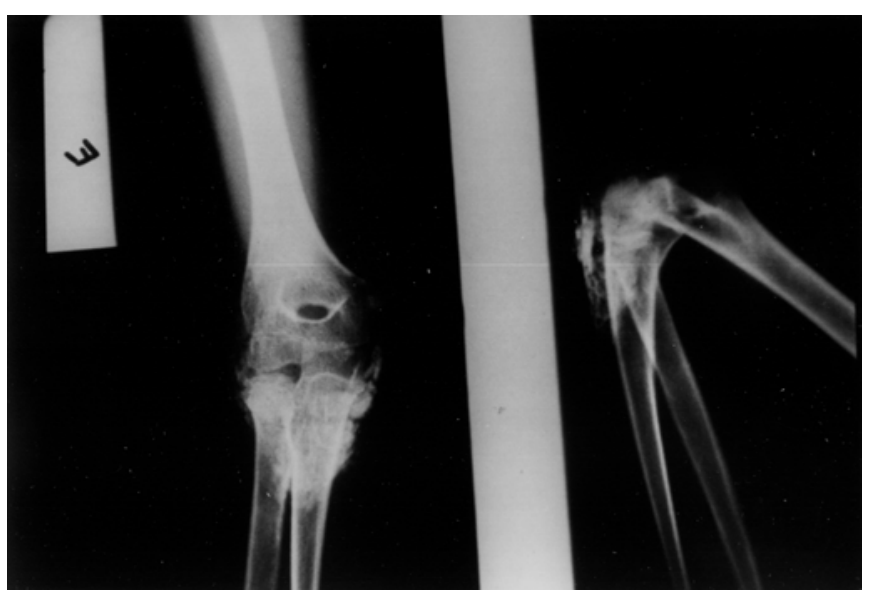

Figura 4 - Calcinose extensa - cotovelos.

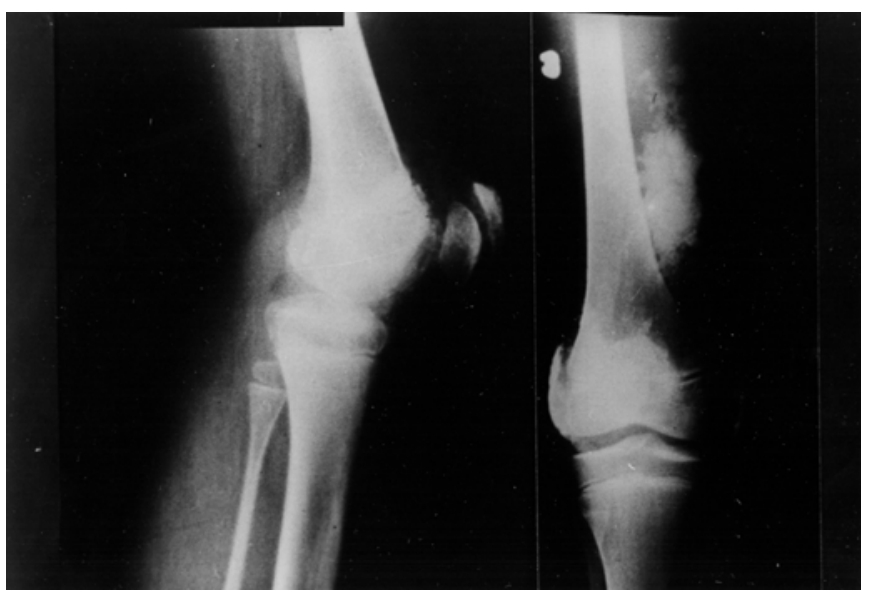

FiguRA 5 - Calcinose extensa - joelhos.

\section{DISCUSSÃO}

A calcinose afeta mais de $70 \%$ dos pacientes com DM juvenil ${ }^{(3)}$ e mais de $45 \%$ dos pacientes com ES, particularmente aqueles com acometimento cutâneo limitado ${ }^{(1)}$. Os nódulos calcificados são compostos por cristais de hidroxiapatita, cristais de oxalato de cálcio, fibras colágenas degeneradas e diversas impurezas, incluindo ácido úrico ${ }^{(3)}$. As lesões estão associadas à inflamação local que causa dor, contraturas articulares e úlceras cutâneas. A distribuição dos nódulos calcificados difere na ES e na DM, embora possam afetar o tecido subcutâneo em qualquer parte do corpo.

Na DM juvenil, o tratamento agressivo da doença parece diminuir a incidência de calcinose ${ }^{(4)}$, porém o tratamento é muito difícil após o estabelecimento da calcinose. Várias drogas têm sido testadas, como a probenecida ${ }^{(5,6)}$, o warfarin $^{(7,8)}$, o diltiazem ${ }^{(9,10)}$, o hidróxido de alumínio $^{(11,12)}$, e a colchicina ${ }^{(13)}$, mas nenhuma delas demonstrou resultados consistentes. Há poucos dados sobre o uso dos bisfosfonatos na calcinose cutânea ${ }^{(4,14,15)}$. A razão para o seu uso reside na hipótese de que parte do cálcio presente nas calcificações seja originada do osso, e na elevada remodelação óssea presente em alguns destes pacientes ${ }^{(3)}$; desta forma, a inibição da reabsorção óssea diminuiria a disponibilidade de cálcio, reduzindo sua deposição nos nódulos calcificados. Os bisfosfonatos também parecem inibir a deposição de cálcio e reduzir a inflamação local, mas seu exato mecanismo de ação ainda não está completamente elucidado.

A paciente apresentou uma significativa e extensa calcinose que não respondeu ao tratamento com colchicina, diltiazem e alendronato sódico, cursando com importante incapacidade funcional. A escolha inicial do alendronato sódico deveu-se à facilidade da sua administração. $\mathrm{O}$ uso subseqüente do clodronato resultou em significativa melhora das manifestações clínicas relacionadas com a inflamação ao redor das calcificações, como a dor e as ulcerações cutâneas. Não houve melhora no número e no tamanho das calcificações, como observado através de radiografias e cintilografias, mas também é verdade que não houve aumento dos depósitos. Considerando a evolução agressiva desta paciente, a estabilização da calcinose já representou uma boa evolução inicial.

É possível que um período mais longo de uso do clodronato possa efetivamente reduzir as calcificações, ou talvez uma dose maior possa ser necessária. A despeito da falha na redução das calcificações, a melhora na qualidade de vida da paciente gerada pelo uso do bisfosfonato foi 
importante o bastante para justificar a manutenção das infusões bimestrais de clodronato. A redução nas infecções cutâneas secundárias de repetição também deve ser levada em conta.

Embora a remissão espontânea da calcinose não seja infreqüente ${ }^{(16,17)}$, não é provável neste caso específico que tenha havido uma coincidência entre a remissão espontânea da calcinose e a introdução do clodronato, uma vez que existiu uma clara relação temporal entre o uso do clodronato

\section{REFERÊNCIAS}

1. Clements PJ, Medsger TA Jr, Feghali CA: Cutaneous involvement in systemic sclerosis. Em Clements PJ, Furst DE (Editors): Systemic sclerosis. $2^{a}$ edição, Filadélfia, Lippincott Williams \& Wilkins, pg. 129-50, 2004.

2. Medsger TA Jr, Oddis CV: Inflammatory muscle disease: Clinical features. Em Klippel JH, Dieppe PA (Editors): Rheumatology. $2^{\mathrm{a}}$ edição, London, Mosby, 7:13.1-14, 1998.

3. Mukamel M, Horev G, Mimouni M: New insights into calcinosis of juvenile dermatomyositis: a study of composition and treatment. J Pediatr 138: 763-6, 2001.

4. Fisler RE: Aggressive management of juvenile dermatomyositis results in improved outcome and decreased incidence of calcinosis. J Am Acad Dermatol 47: 505-11, 2002.

5. Eddy MC, Leelawattana R, McAlister WR, Whyte MP: Calcinosis universalis complicating juvenile dermatomyositis: resolution during probenecid therapy. J Clin Endocrinol Metab 82: 3536-42, 1997.

6. Harel L, Harel G, Korenreich L, Straussberg R, Amir J: Treatment of calcinosis in juvenile dermatomyositis with probenecid: the role of phosphorus metabolism in the development of calcifications. J Rheumatol 28: 1129-32, 2001.

7. Berger RG, Featherstone GL, Raasch RH, McCartney WH, Hadler NM: Treatment of calcinosis universalis with low-dose warfarin. Am J Med 83: 72-6, 1987.

8. Yoshida S, Torikai K: The effects of warfarin on calcinosis in a patient with systemic sclerosis. J Rheumatol 20: 1233-5, 1993. e a melhora clínica, enquanto os depósitos de calcinose estavam crescendo de maneira muito importante antes do início do clodronato.

Em conclusão, atualmente não existe terapêutica comprovadamente eficaz no controle da calcinose na DM e na ES, mas, de acordo com o presente relato, o uso do clodronato endovenoso pode representar uma alternativa para o alívio sintomático e para a cicatrização de úlceras cutâneas em pacientes com extensa e incapacitante calcinose cutânea.

9. Vayssairat M, Hidouche D, Abdoucheli-Baudot N, Gaitz JP: Clinical significance of subcutaneous calcinosis in patients with systemic sclerosis: does diltiazem induce its regression? Ann Rheum Dis 57: 252-4, 1998.

10. Ichiki Y, Akiyama T, Shimozawa N, Suzuki Y, Kondo N, Kitajima Y: An extremely severe case of cutaneous calcinosis with juvenile dermatomyositis and successful treatment with diltiazem. Br J Dermatol 144: 894-7, 2001.

11. Wang $\mathrm{W}$, Lo W, Wong C: Calcinosis cutis in juvenile dermatomyositis: remarkable response to aluminum hydroxide therapy. Arch Dermatol 124: 1721-2, 1988.

12. Nakagawa $\mathrm{T}$, Takaiwa $\mathrm{T}$ : Calcinosis cutis in juvenile dermatomyositis responsive to aluminum hydroxide treatment. J Dermatol 50: 558-60, 1993.

13. Fuchs D, Fruchter L, Fishel B, Holtzman M, Yaron M: Colchicine suppression of local inflammation due to calcinosis in dermatomyositis and progressive systemic sclerosis. Clin Rheumatol 5: 527-30, 1986.

14. Metzger AL, Singer FR, Bluestone R, Pearson CM: Failure of dissodium etidronate in calcinosis due to dermatomyositis and scleroderma. N Engl J Med 291: 1294-6, 1974.

15. Weinstein RS: Focal mineralization defect during dissodium etidronate treatment of calcinosis. Calcif Tissue Int 34: 224-8, 1982.

16. Bowyer SL, Blane CE, Sullivan DB, Cassidy JT: Childhood dermatomyositis: factors predicting functional outcome and development of dystrophic calcification. J Pediatr 103: 882-8, 1983.

17. Ostrov BE, Goldsmith DP, Eichenfeld AH, Athreya BH: Hypercalcemia during the resolution of calcinosis universalis in juvenile dermatomyositis. J Rheumatol 18: 1730-4, 1991. 